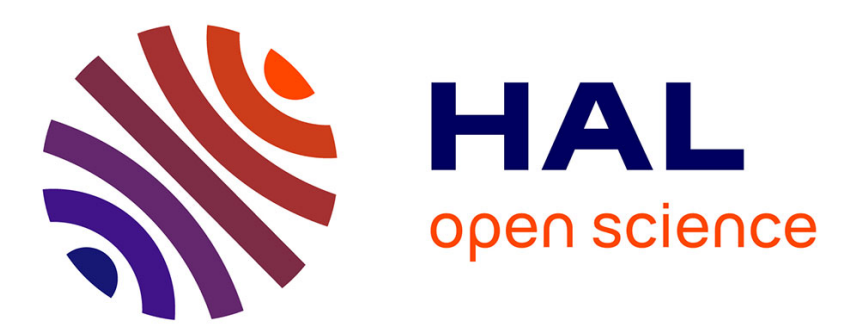

\title{
On Deleterious Mutations in Perennials: Inbreeding Depression, Mutation Load, and Life-History Evolution
}

\author{
Thomas Lesaffre, Sylvain Billiard
}

\section{To cite this version:}

Thomas Lesaffre, Sylvain Billiard. On Deleterious Mutations in Perennials: Inbreeding Depression, Mutation Load, and Life-History Evolution. American Naturalist, 2021, 197 (5), pp.E143-E155. 10.1086/713499 . hal-03260283

\section{HAL Id: hal-03260283 https://hal.science/hal-03260283}

Submitted on 26 Oct 2021

HAL is a multi-disciplinary open access archive for the deposit and dissemination of scientific research documents, whether they are published or not. The documents may come from teaching and research institutions in France or abroad, or from public or private research centers.
L'archive ouverte pluridisciplinaire HAL, est destinée au dépôt et à la diffusion de documents scientifiques de niveau recherche, publiés ou non, émanant des établissements d'enseignement et de recherche français ou étrangers, des laboratoires publics ou privés. 


\title{
On deleterious mutations in perennials: inbreeding depression, mutation load and life-history evolution
}

\author{
Thomas Lesaffre $^{*, 1}$ and Sylvain Billiard ${ }^{2}$ \\ ${ }^{1}$ CNRS, Univ. Lille, UMR 8198 - Evo-Eco-Paleo, F-59000 Lille, France ${ }^{2}$ Univ. Lille, CNRS, \\ UMR 8198 - Evo-Eco-Paleo, F-59000 Lille, France \\ * Corresponding author. E-mail: thomas.lesaffre@univ-lille.fr
}

Description of the manuscript: The main text of the manuscript, excluding captions and headers, is 5912 words long. There are 4 figures in the main text, numbered from 1 to 4 . In the present file, pages 1 to 33 correspond to the main text (including title page, abstract and litterature cited), while the remaining pages (33 to 73) correspond to appendices. There are 5 sections in Appendices, which are all available at the end of the manuscript file. There are 12 figures in Appendices, numbered from S1 to S12.

Keywords: age-structured population, fitness landscape, mutation-selection balance, epistasis, size-structured population.

Manuscript type: Article. 


\begin{abstract}
In Angiosperms, perennials typically present much higher levels of inbreeding depression than annuals. One hypothesis to explain this pattern stems from the observation that inbreeding depression is expressed across multiple life stages in Angiosperms. It posits that increased inbreeding depression in more long-lived species could be explained by differences in the way mutations affect fitness, through the life stages at which they are expressed. In this study, we investigate this hypothesis. We combine a physiological growth model and multilocus population genetics approaches to describe a full genotypeto-phenotype-to-fitness map. We study the behaviour of mutations affecting growth or survival, and explore their consequences in terms of inbreeding depression and mutation load. Although our results only agree with empirical data within a narrow range of conditions, we argue that they may point us towards the type of traits capable of generating high inbreeding depression in long-lived species, that is traits under sufficiently strong selection, on which selection decreases sharply as life expectancy increases. Then, we study the role deleterious mutations maintained at mutation-selection balance may play in the joint evolution of growth and survival strategies.
\end{abstract}




\section{Introduction}

Perennials, which make up the majority of Angiosperms ( 70\%, Munoz et al., 2016), typically present much higher levels of inbreeding depression than annuals. Indeed, metaanalyses found inbreeding depression to span from $\delta \approx 0.2$ on average in short-lived herbaceous species to $\delta \approx 0.5$ in long-lived herbaceous species and shrubs, and $\delta \approx 0.6$ in woody species (Duminil et al., 2009; Angeloni et al., 2011). Inbreeding depression, defined as the reduction in fitness of inbred relative to outbred individuals, is thought to be mainly due to the increased homozygosity of inbred individuals for recessive deleterious mutations segregating at low frequencies in populations (Charlesworth and Charlesworth, 1987; Charlesworth and Willis, 2009). Why inbreeding depression is higher in more longlived species is still poorly understood, despite the potential significance of this pattern for important evolutionary questions, such as mating systems or dispersal rates evolution (Barrett and Harder, 1996; Roze and Rousset, 2005; Epinat and Lenormand, 2009; Duputié and Massol, 2013), and for more applied issues, as many cultivated species are perennial (e.g. fruit trees in general) and efforts are being made to develop perennial grain crops (DeHaan and Van Tassel, 2014).

Two hypotheses have been proposed to explain this pattern. The first hypothesis was formally put forward by Scofield and Schultz (2006). In plants, mutations occurring in somatic tissues may be passed onto the offspring, because they do not have a segregated germline. Thus, Scofield and Schultz (2006) proposed that more long-lived species may accumulate more somatic mutations as they grow and transmit them to their offspring, thereby generating the increase in inbreeding depression observed in such species. Phenotypic data in a long-lived clonal shrub (Vaccinium angustifolium, Bobiwash et al., 2013), 
and genomic results in Quercus robur (Plomion et al., 2018) demonstrated that some somatic mutations can indeed be passed onto the offspring. However, the number of detected heritable somatic mutations is low (Schmid-Siegert et al., 2017; Plomion et al., 2018), and recent studies have concluded that the number of cell divisions from embryonic cells to gametes production may be much lower than previously thought (Burian et al., 2016; Watson et al., 2016; Burian et al., 2016; Schmid-Siegert et al., 2017; Lanfear, 2018), due for instance to early specification and quiescence mechanisms of axillary meristems cells, resulting in little opportunity for heritable mutations to accumulate during plant growth. Furthermore, intraorganismal selection is expected to efficiently purge deleterious somatic mutations, resulting in little to no somatically generated mutation load at the population level (Otto and Orive, 1995). Hence, although somatic mutations can be inherited and contribute to the mutation load in plants, their relative significance compared with meiotic mutations remains unclear (Schoen and Schultz, 2019).

The second hypothesis stems from the observation that inbreeding depression is typically expressed across multiple life stages in Angiosperms (Husband and Schemske, 1996; Winn et al., 2011; Angeloni et al., 2011). It posits that increased inbreeding depression in more long-lived species could be explained by the fact that mutations, regardless of whether they are produced during mitosis or meiosis, may differ in the way they affect fitness in annual and perennial populations, through the life stages at which they are expressed. Most theoretical studies of the mutation load focused on the case of mutations affecting fitness on a strictly linear fitness landscape (that is, fitness is the trait, e.g. Charlesworth et al., 1990; Roze, 2015), or through an abstract trait (or set of traits) under stabilising selection on a gaussian fitness landscape (e.g. Roze and Blanckaert, 2014; 
Abu Awad and Roze, 2018). In these cases, inbreeding depression in annual and perennial populations is not expected to differ (Charlesworth, 1980). On the other hand, the dynamics of mutations affecting other aspects of individuals' life cycle, such as survival or growth, were seldom investigated. Morgan (2001) investigated the dynamics of mutations affecting survival between mating events in a perennial populations. They concluded that inbreeding depression should sharply decrease as life expectancy increases, and even become negative for long-lived species (outbreeding depression). However, Morgan (2001) studied mutations with a strong effect on fitness, and assumed no age-structure, that is, individuals did not differ in fecundity or survival probability with age. Yet, such variations with respect to age in both survival and fecundity are observed in perennials. Indeed, while juveniles typically suffer from very high mortality rates, established individuals tend to experience rather low mortalities with particularly slow senescence (Petit and Hampe, 2006). Furthermore, fecundity usually increases dramatically with age in perennials (Franco and Silvertown, 1996), due to the positive scaling of reproductive output with size in plants (Klinkhamer et al., 1985; Weiner et al., 2009). Mutations slowing their bearer's growth could therefore play a role in generating higher inbreeding depression in more long-lived species, as growth delays impact individuals' fecundities negatively and with varying intensity with respect to age or size. This latter aspect of age-structuration in perennials was, to our knowledge, never tackled theoretically.

The present study aims to study the second hypothesis, that is, we investigate the behaviour of meiotic mutations affecting fitness differently with respect to life-history, putting aside somatic mutations accumulation. Namely, we study meiotic mutations affecting growth or survival in a partially selfing population, in which individuals grow as 
they age and fecundity is proportional to size, but survival between mating events is assumed to not depend on age. We combine a physiological growth model (West et al., 2001) and multilocus population genetics approaches (Barton and Turelli, 1991; Kirkpatrick et al., 2002) in order to describe a full genotype-to-phenotype-to-fitness map, where the fitness landscape emerges from developmental processes instead of being assumed a priori. We study the behaviour of different types of mutations affecting growth or survival, and explore their consequences in terms of inbreeding depression and mutation load (Crow, 1958). Then, we show the role deleterious mutations maintained at mutation-selection balance may play in the joint evolution of growth and survival strategies.

\section{Model outline and methods}

Demography. We consider a large population of diploid hermaphrodites, which may survive from one mating season to another with a probability $S$, assumed to be constant with respect to age and size. If they survive, individuals grow between mating events, following a physiological growth model described briefly in the next paragraph. If they die, juveniles, which are assumed to be produced in large excess compared with the resources available for establishment, are recruited to replace the dead, so that population size is kept constant (FIG. 1). We assume that juveniles are produced each year and do not survive to the next mating season if they are not recruited (no dormancy). Each juvenile has a probability $J$ of being recruited. During reproduction, individuals are assumed to contribute to the gamete pool in proportion to their size (the larger an individual, the larger its contribution to the gamete pool), and to self-fertilise at a fixed rate $\alpha$. 
Growth model. We consider the growth model developed by West et al. (2001). The energy available for growth and maintenance at age $t, B_{t}$, is assumed to scale as a $3 / 4$-power law of body size as a result of allometry (Peters, 1983), so that

$$
B_{t}=B_{0} G_{t}^{3 / 4}
$$

where $B_{0}$ is the basal metabolic rate and $G_{t}$ is body size at age $t$. This energy can be subdivided into the energy required to maintain the existing body, controlled by a maintenance cost $c$, and the energy available to produce new body parts, controlled by a production $\operatorname{cost} \varepsilon$, so that growth is fully described by the following differential equation

$$
\frac{d G_{t}}{d t}=\frac{B_{0}}{\varepsilon} G_{t}^{3 / 4}-\frac{c}{\varepsilon} G_{t}
$$

Under this model, individual size naturally saturates when the energy required to maintain the existing body equals the available energy (FIG. 2a-2b). Further details regarding the growth model are given in Appendix I.

Genetic assumptions. Mutations are assumed to occur at rate $U$ (per haploid genome) at a large number of loci, which recombine at constant rate $0 \leqslant r \leqslant \frac{1}{2}$. In three separate models, we consider mutations affecting three different traits, that is we consider mutations affecting one trait at a time (no pleiotropy). Mutations may affect growth by increasing either their bearer's maintenance cost $(c)$ or production cost $(\varepsilon)$, or they may affect its survival. When mutations affect survival, they are assumed to decrease both their bearer's probability of being recruited as a juvenile $(J)$ and its adult survival probability $(S)$. The 
effect of mutations is denoted $s$, with a dominance coefficient $0 \leqslant h \leqslant \frac{1}{2}$. Loci affect traits multiplicatively, so that for any trait $z(z \in\{c, \varepsilon, S\})$, we have

$$
z=z_{0}(1 \pm s)^{H_{o}}(1 \pm s h)^{H_{e}}
$$

where $H_{o}$ (resp. $H_{e}$ ) is the number of homozygous (resp. heterozygous) mutations born by the considered individual. The \pm sign is used as a general notation to indicate the fact that depending on the trait, deleterious mutations may increase it, as is the case for the maintenance and the production cost; or decrease it, as is the case for survival. Note that $s$ is the phenotypic effect of mutations here, not to be confused with $\bar{s}_{z}$ and $\hat{\bar{s}}_{z}$, the selection coefficients associated with mutations which will be defined in the following.

\section{Approximation of the expected number of mutations, inbreeding depression}

and mutation load. Our analytical model is built using the framework introduced by Barton and Turelli (1991) and generalized in Kirkpatrick et al. (2002). In this framework, the genetic dynamic of the population is described using allelic frequencies and genetic associations instead of genotypic frequencies. To do so, we define $X_{i}$ and $\stackrel{*}{X_{i}}$ as the indicator variables associated with the paternally and maternally inherited allele at the $i^{\text {th }}$ locus, respectively. These variables are worth 1 if the deleterious allele is present at the considered position, and 0 otherwise. Thus, Equation (3), which depicts the phenotypic effect of mutations, can be rewritten in terms of these variables as

$$
z=z_{0} \times \prod_{i}\left(1 \pm \operatorname{sh}\left(X_{i}+\stackrel{*}{X_{i}}\right) \pm s(1-2 h) X_{i} \stackrel{*}{X}_{i}\right)
$$


Indicator variables can in turn be used to define centered variables $\zeta_{i}$ and $\stackrel{*}{\zeta}$, which are given by

$$
\zeta_{i}=X_{i}-\mathbb{E}\left[X_{i}\right]=X_{i}-p_{i}, \text { and } \stackrel{*}{\zeta}_{i}=\stackrel{*}{X}_{i}-\mathbb{E}\left[\stackrel{*}{X}_{i}\right]=\stackrel{*}{X}_{i}-p_{i}
$$

By definition, we have $\mathbb{E}\left[\zeta_{i}\right]=\mathbb{E}\left[\stackrel{*}{\zeta_{i}}\right]=0$, and expectations of products of these variables can be used to quantify how the population deviates from Hardy-Weinberg's equilibrium, that is genetic associations in the sense of Barton and Turelli (1991), such as excesses in homozygotes, or linkage and identity disequilibria between loci for instance (see Appendix II.1 for details).

In order to obtain an analytical prediction of the average number of mutations per haploid genome at mutation-selection equilibrium, one needs to find the point at which the rate at which mutations enter the population through mutation during meiosis equals the rate at which mutations are removed from the population by selection. This can be done by following the change in allelic frequencies at selected loci between two timesteps, $\Delta p_{i}$, and looking for the point at which we have $\Delta p_{i}=0$. We do so using two different approaches. In the first approach, we make the assumption that selective pressures acting on mutations at various life-stages can be summarised into a single lifetime fitness expression, so that the population can be studied as an adequately rescaled annual population (the Lifetime Fitness approach, LF - Appendix III.1 and FIG. S1). For each type of mutation, this approach allows us to gain insights into the way selection acts on mutations, by summarising it into a single lifetime selection coefficient, $\bar{s}_{z}$, where $z=c, \varepsilon$ or $S$ depending on the considered trait (not to be confused with $s$, the phenotypic effect of mutation. See Appendix III.1.1 for details). Besides, reasoning in terms of lifetime fitness is paramount to compute key quantities such as inbreeding depression or the mutation load. However, 
the LF approach fails to account for genetic associations correctly because it assumes that all selection occurs before reproduction at each timestep (see FiG. S1), so that it overlooks the fact that selection may occur both in parents and juveniles, that is before and after reproduction, respectively, which induces different effects of selection on genetic associations in these two subpopulations. Thus, in order to obtain approximations of the expected number of mutations per haploid genome accounting for genetic associations, we also use a second approach where we study each step of the life cycle successively (the Life Cycle approach, LC - Appendix III.2 and FIG. S2). We assume that the phenotypic effect of mutations is weak and that the number of segregating mutations is large, following the work of Roze (2015) that we adapted to the case of many mutations affecting a trait rather than fitness directly, in an age- and size-structured population.

Simulations. In order to assess the validity of our analytical approximations, individualcentered simulations were run assuming diploid individuals depicted by two linear chromosomes of length $\lambda$ (in $\mathrm{cM}$ ), along which mutations occur stochastically during meiosis (Roze and Michod, 2010). The number of mutations occurring is sampled from a Poisson distribution with mean $U$, and their position on chromosomes are sampled from a uniform distribution. Recombination is modeled by exchanging segments between chromosomes. Similar to mutations, the number of crossing-overs is sampled from a Poisson distribution with mean $\lambda$, while their positions along chromosomes are randomly drawn in a uniform distribution. The population has a constant size $N$. At each timestep, an individual survives with probability $S$, which can depend on its genotype in the case of mutations affecting survival. If the individual does survive, it grows deterministically depending on its age and individual physiological growth costs (FIG. 2, Equation A5 in Appendix I). If 
it does not, it is replaced by an offspring generated from the parental population, which includes the dead individual, and in which parents are chosen with a probability proportional to their size. The offspring is produced by self-fertilisation with probability $\alpha$, and by random mating otherwise. We measure the average of the trait affected by mutations, the average number of mutations per haploid genome in the population, and inbreeding depression. Inbreeding depression is measured as the relative difference in lifetime reproductive success of selfed and outcrossed individuals. Individuals lifetime reproductive success is obtained by counting the number of times they are chosen as parents before they die. The mutation load in the sense of Crow (1958), that is the decrease in mean fitness of the population compared with a population with no mutations, is measured using the population average of the trait affected by mutations. This average is used to compute the expected mean lifetime fitness in the population (Equation A35 in Appendix III.1.1). Then, this quantity is compared to the expected mean lifetime fitness when mutations are absent. Five replicates were run for each parameter set showing a very small standard deviation, indicating that our simulations are representative of the average behaviour. All programs are available from GitHub (links are available in the Data and Code Accessibility Statement section).

\section{Results}

In what follows, results will be presented as functions of the life expectancy of the population, $E$, that is the average number of reproductive cycles an individual is expected to live through before dying. Since survival is not assumed to vary with respect to age nor size, the probability of an average individual to survive up to exactly age $k$ then die 
follows a geometric distribution of parameter $\bar{S}$, where $\bar{S}$ is the mean survival probability in the population, which is simply $\bar{S}=S$ when mutations affect growth, and depends on mutations segregating in the population when said mutations affect survival. Thus, we have

$$
E=\frac{1}{1-\bar{S}}
$$

\section{Mutation-selection equilibrium}

To study the change in allelic frequencies at selected loci, one needs to quantify the intensity of selection acting on mutations. Generally speaking, the intensity of selection depends on how mutations modify the fitness of their bearer, and how the fitness of their bearer then compares with the fitnesses of all the individuals they are competing with, that is the distribution of fitness in the population. Hence, the intensity of selection faced by a mutation segregating at a given locus may depend on the presence of mutations at other loci, since said mutations may alter both the distribution of fitness in the population and how a mutation at the considered locus affects its bearer's fitness. This second aspect is referred to as epistasis. There are numerous ways by which epistasis may be generated between loci (Phillips, 2008), which can roughly be split into two categories: genotype-to-phenotype epistasis on the one hand, and phenotype-to-fitness epistasis on the other hand. Genotype-to-phenotype epistasis refers to loci which influence each other's expression in the determination of a phenotype, say genes involved in a common metabolic pathway, while phenotype-to-fitness epistasis refers to situations where loci, even if they influence phenotype independently, influence each other because the way the phenotype 
affects fitness is non-linear.

In our model, we assume that mutations affect a phenotype multiplicatively, so that genotype-to-phenotype epistasis does not occur. On the other hand, we do not impose any constraint on the shape of the fitness landscape. Instead, we let the genotype-tophenotype-to-fitness map arise from biological assumptions. The resulting fitness landscape is non-linear, convex, and has singularities at the optima (Equation A35 in Appendix III.1, FIG. S3). Thus, phenotype-to-fitness epistatic interactions are generated, and have to be accounted for to obtain accurate mutation-selection balance approximations, so that quantifying the intensity of selection acting on a mutation at a given locus requires accounting for the average number of mutations it may be in epistatic interaction with. This can be done by following the change in the trait average as mutations segregate (Equation 7, see Appendices III.1.2 and III.2.2 for the derivation of this result under the Lifetime Fitness and the Life Cycle approach, respectively). We find this trait average at mutation-selection balance, when the change in allele frequency is zero.

$$
\left\{\begin{array}{l}
\Delta p_{i}(\bar{z})=0 \\
\bar{z}\left(p_{i}\right)=z_{0} \times \exp \left(\sum_{i} \bar{Z}_{i}\right) \times\left(1+\sum_{j \neq i} \frac{\overline{\Delta Z_{i} \times \Delta Z_{j}}}{2}\right) .
\end{array}\right.
$$

In the first line of Equation (7), $\Delta p_{i}$ is the change in the frequency of the mutant allele at the $i^{\text {th }}$ locus, which depends on the trait average $\bar{z}$. On the second line, $\bar{z}$ is the average of the trait in the population, which depends on the number of mutations per haploid genome. This number of mutations is obtained by summing allelic frequencies over every relevant loci, that is $n=\sum_{i} p_{i}$ (Kimura and Maruyama, 1966). On this second line, the \pm sign indicates the fact that deleterious mutations may increase the average trait of the 
population, as it is the case for the maintenance and the production cost, or decrease it, as it is the case for survival. The term $\sum_{i} \bar{Z}_{i}$ quantifies the effect on the trait of the $n$ mutations per haploid genome borne on average by individuals, neglecting the effects of genetic associations between loci, while the term $\overline{\Delta Z_{i} \times \Delta Z_{j}}$ quantifies the effect of pairwise associations between loci on the trait average. Associations of higher order are neglected. Solving Equation (7) for $p_{i}$ and $\bar{z}$ allows us to obtain predictions for the average of the trait and for the average number of mutations per haploid genome maintained at mutation-selection equilibrium, which we then use to compute the inbreeding depression and mutation load expected at equilibrium.

\section{Fitness effect of mutations neglecting genetic associations}

Under the Lifetime Fitness approach, to leading order in $s$ so that genetic associations between loci can be neglected, Equation (7) simplifies into

$$
\left\{\begin{array}{l}
U+\bar{s}_{z}(\bar{z})\left(h \sum_{i} p_{i}+(1-h) F \sum_{i} p_{i}\right)=0 \\
\bar{z}\left(p_{i}\right)=z_{0} \times \exp \left[ \pm s\left(2 h \sum_{i} p_{i}+(1-2 h) F \sum_{i} p_{i}\right)\right]
\end{array}\right.
$$

where $F=\frac{\alpha}{2-\alpha}$ is the equilibrium inbreeding coefficient. The derivation of this result is explained in Appendix III.1.2 (Equation A44). The first line of Equation (8) shows that when we neglect genetic associations between loci, the selective pressures acting on mutations are encapsulated in a single lifetime selection coefficient $\bar{s}_{z}$, which is specific to each model and emerges from the application of the Taylor expansion methods presented in Appendix II.2.1 to lifetime fitness. Its expression for each type of mutation studied is given in Appendix III.1.1. 
The phenotypic effect of each type of mutation (FIG. 2, top row), and the resulting leading order lifetime selection coefficients are presented in FIG. 2 (bottom row). These coefficients are all negative because we consider deleterious mutations, and depend on the population average of the trait, owing to the epistatic mechanisms described in the former section. Although it is not possible to fully disentangle the effects of epistasis from the effects of direct selection at the locus, one may gain insight into the sign of epistasis by studying the direction in which $\bar{s}_{z}$ coefficients are changed when the number of segregating mutations increases in the population. The $\bar{s}_{z}$ coefficients plotted in black were obtained assuming mutations are absent (i.e. $\bar{z}=z_{0}$ ), while those plotted in red were obtained assuming $n=\sum_{i} p_{i}=10$ mutations were segregating at surrounding loci. In other words, the coefficients plotted in black in Fig. 2 (bottom row) represent the intensity of selection a mutation would face if a single locus was modeled, while the coefficients plotted in red depict the intensity of selection a mutation would face when in interaction with ten mutations at other loci.

Mutations affecting the maintenance cost $c$ cause size differences to increase as individuals age (FIG. 2a). Hence, their fitness effect increases with life expectancy (FIG. 2d). Furthermore, epistatic interactions cause selection against mutations to increase that is, we observe negative epistasis. On the contrary, mutations affecting the production cost $\varepsilon$ do not affect individuals' maximal size, as they asymptotically tend to the same size, but rather the speed at which they reach it (FIG. 2b). Therefore, the growth delay mutated individuals accumulate in early years fades away in older individuals. This causes selection against mutations affecting $\varepsilon$ to decrease with respect to life expectancy, because they become gradually neutral in older age-classes. However, epistasis also causes selection 
against mutations to increase, so that it is negative in this case as well.

Mutations affecting survival cause individuals to perform less mating events in a lifetime. They also cause mutated individuals to perform less well during mating events, because they tend to be younger than unmutated individuals (FIG. 2c). Thus, agestructure increases selection against mutations affecting survival. Furthermore, selection against mutations affecting survival strongly increases as life expectancy increases (FIG. 2f), contrary to mutations affecting growth costs, whose fitness effects remain moderate (FIG. 2d-e). Besides, life expectancy decreases as the number of segregating mutations increases (because the mean survival probability $\bar{S}$ decreases), which reduces the intensity of selection acting on mutations, thereby generating strong positive epistasis (FIG. 2f, in this panel the red and black lines overlie because the trait affected by mutations is plotted on the x-axis, contrary to mutations affecting growth).

Overall, the results described in FIG. 2 show that mutations affecting different traits on the same genotype-to-phenotype-to-fitness map face very different selective pressures, both in magnitude and in the way they vary with life expectancy.

\section{Average number of mutations, inbreeding depression and mutation load}

The intensity of selection acting on mutations does not depend on the values of $c$ and $\varepsilon$, but on the ratio $\frac{c}{\varepsilon}$ (Appendix III.1.1). Thus, in what follows, results are described using this ratio. FiguRE 3 presents results for the average number of mutations per haploid genome maintained at equilibrium, and the resulting inbreeding depression and mutation load for $h=0.25$ and $\frac{c}{\varepsilon}=1$ (other parameter sets are shown in appendix as results are qualitatively similar, FIG. S5 to S12). Analytical predictions, which are depicted by solid 
lines, are obtained by solving Equation (7) numerically using the LC approach accounting for pairwise genetic associations (Appendix III.2), and dots depict simulations results. Results obtained with the LF approach (lighter lines, Appendix III.1) are also presented on the first row of Fig. 3 .

The first row in Fig. 3 presents the log-scaled average number of mutations per haploid genome maintained at mutation-selection balance. For mutations affecting the maintenance cost and survival, the average number of mutations per haploid genome maintained at equilibrium $\left(n=\sum_{i} p_{i}\right)$ decreases as life expectancy increases (FIG. 3a and 3c), because selection against mutations increases. This effect is more marked for mutations affecting survival because selection is stronger in this case (FIG. 2d and 2f). Conversely, $n$ increases as life expectancy increases for mutations affecting the production cost, because selection against these mutations weakens as life expectancy increases (FIG. 2e). In every case, $n$ decreases as the selfing rate increases due to the purging effect of self-fertilisation. The LF approach performs just as well as the LC approach for mutations affecting growth costs, but tends to fail for mutations affecting survival due to the fact that it overlooks important aspects of the model, as discussed above.

The middle row in Fig. 3 shows the levels of inbreeding depression generated by mutations maintained at mutation-selection balance. Large differences in the number of deleterious mutations maintained per genome do not translate into strong variations in inbreeding depression with respect to life expectancy. However, variations are still observed and differ between mutation types. Indeed, inbreeding depression always decreases with life expectancy when mutations affect survival, irrespective of the selfing rate (FIG. 3f), contrary to mutations affecting growth. Mutations affecting $c$ generate slightly higher 
inbreeding depression in more short-lived species at low selfing rate, but this pattern is reversed at higher selfing rates (FIG. 3d). Conversely, mutations affecting $\varepsilon$ generate higher inbreeding depression in more long-lived species for low $\alpha$ and this pattern is reversed for high $\alpha$ (FIG. 3e). This is the result of the interaction between life expectancy, the shape of the fitness landscape and selfing, which is difficult to disentangle as these elements interact in non-trivial ways. Indeed, the shape of the fitness landscape changes with respect to life expectancy, which causes variations in the intensity of selection and epistatic interactions, and selfing changes the distribution of fitness by altering homozygosity and by promoting the purging of deleterious mutations in doing so, so that it too alters epistatic interactions.

The bottom row in Fig. 3 presents the mutation load measured in the sense of Crow (1958) which results from the mutations segregating at mutation-selection balance. Intuitively, one would assume that having more deleterious mutations segregating in the population should lead to a higher mutation load. Yet, the mutation load is lower when mutations are more numerous for all three mutation types in our model. Importantly, this result is not a mere reflection of differences in the absolute strength of selection acting on mutations in populations with different life expectancies. Otherwise, changing the phenotypic effect of mutations in a given model and for a given parameters set would also change the mean phenotypic deviation from the optimum, and therefore the mutation load. This is not what we observe. Indeed, Figure S4 in Appendix V shows that when the effect of mutations is made ten times larger, this does not cause differences in the equilibrium phenotypic deviation, although the number of segregating mutations at equilibrium was considerably lower (because selection was stronger). This observation is consistent with results obtained by previous authors (Bataillon and Kirkpatrick, 2000), who showed that 
when the population exceeds a particular size, the mutation load becomes independent from the strength of selection acting on mutations. We argue that the differences we observe in terms of mutation load are imputable to differences in the shape of the fitness landscapes (FIG. S3), which are not fully captured by differences in the efficacy of selection.

\section{Consequences for life-history evolution}

The mutations segregating in the population cause the mean phenotype to deviate from its initial value. As the intensity of selection acting on mutations depends on this mean phenotype, this leads to a joint equilibrium for both the mean phenotype and the number of mutations segregating in the population (Appendices II and III). This equilibrium varies with respect to life expectancy and mating system. Increasing the selfing rate slightly decreases the deviation of the mean phenotype from its initial value, because selfing induces a better purging of mutations. More importantly, the equilibrium phenotypes vary significantly between populations with different life expectancies. Indeed, as species become more long-lived, the equilibrium maintenance cost decreases, so that maximal size increases, and the equilibrium production cost increases, so that growth is slowed down. This means that if, for any reason, life expectancy changes in a species, the growth strategy should also be changed as a consequence of the selective pressures acting on deleterious mutations maintained at mutation-selection balance being altered. 


\section{Discussion}

Inbreeding depression. In this paper, we set off to study the magnitude and variation of inbreeding depression generated by mutations affecting survival or growth in relation to life expectancy. We showed that inbreeding depression at mutation-selection balance varies weakly with respect to life expectancy when mutations affect growth, while inbreeding depression decreases more sharply as life expectancy increases in the case of mutations affecting survival. We showed that these differences between mutation types can be attributed to differences in the intensity of selection and how it varies with respect to life expectancy. In any case, our results only agree with empirical data in a limited region of the parameters space, that is when selfing is rare and selective pressures acting on mutations decrease as life expectancy increases, as it is the case for mutations affecting the production cost most noticeably. Although these results may at first glance seem to point to a more preponderant role for somatic mutations accumulation in generating the sharp increase in inbreeding depression observed in more long-lived species, we argue in what follows that they may rather point us towards the type of traits mutations should affect in order to produce this empirically observed pattern, irrespective of the way mutations are produced.

As stated above, differences in the magnitude of inbreeding depression with respect to life expectancy were quantitatively small in every investigated case when mutations affected growth, that is either the maintenance or the production cost, even for low dominance coefficients and high mutation rates. This result can be explained by the moderate variations in the intensity of selection on growth-related traits with respect to life expectancy, and by the fact that we only considered mutations with weak phenotypic effects, 
which in this case translated into weak fitness effects. Therefore, this result is consistent with previous population genetics work that showed that inbreeding depression becomes independent of the intensity of selection when selection is weak and the population is large (e.g. Charlesworth et al., 1990; Bataillon and Kirkpatrick, 2000; Roze, 2015). As for mutations affecting survival, their dynamics were studied by Morgan (2001) prior to this study in a perennial, but not age-structured population. They concluded that inbreeding depression should quickly decrease as life expectancy increases, and that significant outbreeding depression should be observed in long-lived species. They argued this result could be attributed to the greater variance in fitness observed among the offspring produced by self-fertilisation, which led to a higher mean lifetime fitness among them. Although they did not state it explicitly, this result stems from the fact that a very small number of mutations were maintained at mutation-selection balance in long-lived species under the parameter sets they investigated. Indeed, Morgan (2001) considered large phenotypic effects of mutations ( $s=0.1$ and $s=1.0$ ), which resulted in tremendous lifetime fitness effects and therefore in the maintenance of almost no mutations at equilibrium. This led variance in fitness to dominate over other aspects. Here, we investigated mutations with much lower phenotypic effects $(s=0.005)$, and did not observe the same patterns. In fact, we show that although inbreeding depression still decreases with life expectancy, this decrease is considerably reduced, and outbreeding depression is no longer observed. Thus, if substantial inbreeding depression is observed on traits related to survival in long-lived species, we conclude that this should be caused by mutations with very weak phenotypic effects, or whose effects are limited to early stages of life, as mutations would otherwise not be maintained in the population. 
Taken together, our results and those of Morgan (2001) show that variations in the fitness effect of mutations with respect to life expectancy can generate differences in inbreeding depression, provided that these variations are strong enough. Furthermore, we showed that inbreeding depression maintained at mutation-selection balance decreases when the fitness effect of mutations increases, as said mutations are more efficiently purged from the population. This implies that mutations facing sufficiently strong selective pressures, which decrease sharply as life expectancy increases, could in principle generate the increase in inbreeding depression observed in more long-lived species. In the light of this result, we propose that asking on what traits selection is expected to be strong and to decrease significantly as life expectancy increases in plants is a track worth following in order to further our understanding of the mechanisms underlying inbreeding depression. Importantly, this result is not in contradiction with the somatic mutations accumulation hypothesis formulated by Scofield and Schultz (2006). Indeed, the intensity of selection acting on mutations matters for their persistence in the population and for the magnitude of inbreeding depression generated by them, regardless of the way mutations are produced.

Mutation load. Contrary to what one might intuitively expect, we showed that more mutations maintained at mutation-selection balance led to a lower mutation load in all three of our models, and that this result is not a straightforward consequence of differences in the intensity of selection acting on mutations, in agreement with results obtained by previous authors (Bataillon and Kirkpatrick, 2000). Instead, it is a consequence of differences in the shape of fitness landscapes between species with different life expectancies on the one hand, and of the interaction between the mean phenotype and the intensity of selection on the other hand. This result highlights the fact that incorporating a phe- 
notypic dimension to population genetics studies may lead to counter-intuitive results. In particular, when fitness landscapes are obtained as the result of biological assumptions, and not arbitrarily assumed to be of a particular shape, unusual interactions with various aspects of species life-histories may result in novel predictions. For instance, in the present study, we showed that the mutation load may behave very differently depending on the trait affected by mutations and life expectancy. Therefore, we conclude that comparing mutation loads between species with contrasting life-histories may sometimes be misleading, and would likely require trait-specific approaches.

Epistasis emerging from the fitness landscape. The phenotypic dimension of the genotype-to-phenotype-to-fitness map is usually overlooked in mutation load dynamics studies. Indeed, most theoretical investigations interested in such matters consider mutations affecting fitness multiplicatively (e.g. Kondrashov, 1985; Charlesworth et al., 1990; Roze, 2015), so that fitness is the phenotype affected by mutations and the phenotype-tofitness map (the fitness landscape) is strictly linear. Hence, neither phenotype-to-fitness epistasis nor genotype-to-phenotype epistasis occur in these models. Some authors however considered the effects of epistasis on mutation load dynamics.

Charlesworth et al. (1991) studied uniformly deleterious mutations under synergistic selection. Because they assumed mutations to affect fitness directly too, no phenotype-tofitness epistasis occured in their model. On the other hand, genotype-to-phenotype epistasis occured because they assumed mutations to affect fitness synergistically. Abu Awad and Roze (2019) generalised Charlesworth et al. (1991)'s results, showing that their model is equivalent to assuming fixed negative pairwise epistasis between loci. Furthermore, they showed that different forms of pairwise epistasis have different effects: while additive-by- 
additive epistasis lowers the frequency of mutations by increasing selection, additive-bydominance and dominance-by-dominance epistasis tend to increase inbreeding depression by making homozygotes less fit.

Phenotype-to-fitness epistasis was also considered in the case of mutations affecting an abstract trait (or set of traits) under stabilising selection (e.g. Abu Awad and Roze, 2018, 2019). These studies assumed mutations to affect the trait(s) additively, so that no genotype-to-phenotype epistasis occured, but assumed the fitness landscape to be gaussian or quasi-gaussian (Fisher, 1930), so that phenotype-to-fitness epistasis occured as the landscape was non-linear. However, because the fitness landscape was symmetrical with respect to the optimal phenotype and mutations occured in both directions at the same rate, epistasis was null on average (Gros et al., 2009). Yet, it influenced mutation load dynamics through variance effects, by generating associations between alleles with compensatory effects, and decreasing the efficiency of selection on deleterious alleles when these associations stay moderate.

In this paper, we studied the dynamics of mutations affecting growth and survival using a physiological growth model (West et al., 2001). Hence, the fitness effect of mutations emerged from biological assumptions instead of being assumed a priori. The resulting fitness landscape had singularities at the optimal phenotypes, as in the absence of tradeoffs, immortality $(S=1)$ is the optimal survival strategy and leads to an infinite life expectancy, and null growth $\operatorname{costs}(c=\varepsilon=0)$ are the optimal growth strategy, which leads to infinitely large individuals. Furthermore, these optimal phenotypes were situated on the boundaries of the landscape for all three traits, as negative growth costs or survival probabilities greater than one are meaningless. As a consequence, mutations were 
always under directional selection in our model. We hence focused on the case of mutations increasing growth costs or decreasing survival, that is deleterious mutations, as opposite mutations would always be favoured and eventually reach fixation. Here, the fitness landscape was non-linear (FIG. S3). Hence, although we assumed mutations to affect phenotypes multiplicatively, so that no genotype-to-phenotype epistasis occured, phenotype-to-fitness epistasis was generated. Contrary to models assuming stabilising selection (e.g. Abu Awad and Roze, 2018, 2019), epistasis was non-zero on average in our model, and its direction (positive or negative) differed depending on the phenotype affected by mutations. It was however difficult to isolate the effects of epistasis in our model because pairwise epistatic interactions are not sufficient to capture these effects, as considering only pairwise interactions is equivalent to linearising the effect of mutations on the trait average, which only works when very few mutations segregate in the population.

Life-history evolution. Plants vary widely in life expectancy and stature, with life expectancies ranging from a few weeks to hundreds, possibly thousands of years, and stature spanning several orders of magnitude across Tracheophytes (Ehrlén and Lehtilä, 2002). These variations are correlated. Indeed, long-lived species tend to grow slower than shortlived species (Salguero-Gómez et al., 2016). In life-history traits evolution theory, this type of correlation is usually interpreted in terms of trade-offs, with populations evolving towards the evolutionarily stable allocation of resources between growth, survival and reproduction, given a number of constraints (Stearns, 1992). In this paper, we have shown that the equilibrium maintenance and production costs differ between life expectancies, causing more long-lived species to grow slower but ultimately larger than more short-lived species, as commonly expected. However, the mechanism underlying this result is com- 
pletely different. Indeed, in our model, life-history traits do not evolve jointly in response to trade-offs. Instead, the equilibrium growth costs are modified when life expectancy varies because the selective pressures acting on the many deleterious mutations affecting these traits are altered, leading to a more or less efficient purging of said mutations and thereby phenotypic differences at mutation-selection equilibrium. Thus, our results suggest that life-history traits may sometimes evolve jointly regardless of trade-offs, because a change in a given trait may alter the efficiency of purging of deleterious mutations affecting other traits.

\section{Acknowledgements}

The authors thank Sylvain Glémin, Florence Débarre, Vincent Castric and Denis Roze for taking the time to discuss this work. This work was funded by the European Research Council (NOVEL project, grant \#648321). The authors also thank the Région Hautsde-France, and the Ministère de l'Enseignement Supérieur et de la Recherche (CPER Climibio), and the European Fund for Regional Economic Development for their financial support. Finally, the authors would like to thank the Editor and Associate Editor along with the both anonymous reviewers for the time and effort they devoted to the improvement of this article.

\section{Author Contribution Statement}

Both authors contributed to the conceptualization of the work presented in this article. Mathematical developments and individual-centered simulations were carried out by TL. Both authors contributed to the analysis of the results and to writing and editing the 
manuscript. SB supervised the work and acquired the funding.

\section{Data and Code Accessibility Statement}

All individual-centered simulation and numerical computation programs are available on

GitHub (https://github.com/Thomas-Lesaffre/On_deleterious_mutations_in_perennials).

\section{References}

Abu Awad, D., and D. Roze. 2018. Effects of partial selfing on the equilibrium genetic variance, mutation load and inbreeding depression under stabilizing selection. Evolution $72: 751-769$

2019. Epistasis, inbreeding depression and the evolution of self-fertilization. Evolution .

Angeloni, F., N. Ouborg, and R. Leimu. 2011. Meta-analysis on the association of population size and life history with inbreeding depression in plants. Biological Conservation 144:35-43.

Barrett, S. C., and L. D. Harder. 1996. The comparative biology of pollination and mating in flowering plants. Philosophical Transactions of the Royal Society of London B 351:1271-1280.

Barton, N., and M. Turelli. 1991. Natural and sexual selection on many loci. Genetics $127: 229-255$. 
Bataillon, T., and M. Kirkpatrick. 2000. Inbreeding depression due to mildly deleterious mutations in finite populations: size does matter. Genetics Research 75:75-81.

Bobiwash, K., S. Schultz, and D. Schoen. 2013. Somatic deleterious mutation rate in a woody plant: estimation from phenotypic data. Heredity 111:338-344.

Burian, A., P. Barbier de Reuille, and C. Kuhlemeier. 2016. Patterns of stem cell divisions contribute to plant longevity. Current Biology 26:1385-1394.

Charlesworth, B. 1980. Evolution in age-structured populations. Cambridge Studies in Mathematical Biology.

Charlesworth, B., M. Morgan, and D. Charlesworth. 1991. Multilocus models of inbreeding depression with synergistic selection and partial self-fertilization. Genetics 57:177-194.

Charlesworth, D., and B. Charlesworth. 1987. Inbreeding depression and its evolutionary consequences. Annual Review of Ecology and Systematics 18:237-268.

Charlesworth, D., M. Morgan, and B. Charlesworth. 1990. Inbreeding depression, genetic load, and the evolution of outcrossing rates in a multilocus system with no linkage. Evolution 44:1469-1489.

Charlesworth, D., and J. Willis. 2009. The genetics of inbreeding depression. Nature reviews genetics 10:783-796.

Crow, J. 1958. Some possibilities for measuring selection intensities in man. Human Biology 61:763-775.

DeHaan, L., and D. Van Tassel. 2014. Useful insights from evolutionary biology for developing perennial grain crops. American Journal of Botany 101:1801-1819. 
Duminil, J., O. Hardy, and R. Petit. 2009. Plant traits correlated with generation time directly affect inbreeding depression and mating system and indirectly genetic structure. BMC Evolutionary Biology 9:177.

Duputié, A., and F. Massol. 2013. An empiricist's guide to theoretical predictions on the evolution of dispersal. Interface Focus 40:20130028.

Ehrlén, J., and K. Lehtilä. 2002. How perennial are perennial plants? Oikos 98:1070-1072.

Epinat, G., and T. Lenormand. 2009. The evolution of assortative mating and selfing with in- and outbreeding depression. Evolution 63:2047-2060.

Fisher, R. 1930. The Genetical Theory of Natural Selection. Oxford University Press.

Franco, M., and J. Silvertown. 1996. Life-history variation in plants: an exploration of the fast-slow continuum hypothesis. Philosophical Transactions of the Royal Society of London B 351:1341-1348.

Gros, P.-A., H. Le Nagard, and O. Tenaillon. 2009. The evolution of epistasis and its links with genetic robustness, complexity and drift in a phenotypic model of adaptation. Genetics 182:277-293.

Husband, B., and D. W. Schemske. 1996. Evolution of the magnitude and timing of inbreeding depression in plants. Evolution 50:54-70.

Kimura, M., and T. Maruyama. 1966. The mutation load with epistatic gene interactions in fitness. Genetics 54:1337-1351.

Kirkpatrick, M., T. Johnson, and N. Barton. 2002. General models of multilocus evolution. Genetics 161:1727-1750. 
Klinkhamer, P., E. Meelis, T. De Jong, and J. Weiner. 1985. On the analysis of sizedependent reproductive output in plants. Functional Ecology 6:308-316.

Kondrashov, A. 1985. Deleterious mutations as an evolutionary factor. II. facultative apomixis and selfing. Genetics 111:635-653.

Lanfear, R. 2018. Do plants have a segregated germline? PloS Biology 16.

Morgan, M. 2001. Consequences of life history for inbreeding depression and mating system evolution in plants. Proceedings of the Royal Society of London B 268:1817-1824.

Munoz, F., C. Violle, and P.-O. Cheptou. 2016. CSR ecological strategies and plant mating systems: outcrossing increases with competitiveness but stress-tolerance is related to mixed mating. Oikos 125:1296-1303.

Otto, S., and M. Orive. 1995. Evolutionary consequences of mutation and selection within an individual. Genetics 141:1173-1187.

Peters, R. 1983. The ecological implications of body size. Cambridge studies in ecology.

Petit, R., and A. Hampe. 2006. Some evolutionary consequences of being a tree. Annual Review of Ecology, Evolution, and Systematics 37:187-214.

Phillips, P. 2008. Epistasis - the essential role of gene interactions in the structure and evolution of genetic systems. Nature Reviews Genetics pages 855-867.

Plomion, C., J.-M. Aury, J. Amselem, T. Leroy, F. Murat, S. Duplessis, S. Faye, N. Francillonne, K. Labadie, G. Le Provost, I. Lesur, J. Bartholomé, P. Faivre-Rampant, A. Kohler, J.-C. Leplé, N. Chantret, J. Chen, A. Diévrat, T. Alaeitabar, V. Barbe, 
C. Belser, H. Bergès, C. Bodénès, M.-B. Bogeat-Triboulot, M.-L. Bouffaud, B. Brachi, E. Chancerel, D. Cohen, A. Couloux, C. Da Silva, C. Dossat, C. Ehrenmann, F. Gaspin, J. Grima-Pettenati, E. Guichoux, A. Hecker, S. Herrmann, P. Hugueney, I. Hummel, C. Klopp, C. Lalanne, M. Lascoux, E. Lasserre, M.-L. Lemainque, A. Desprez-Loustau, I. Luyten, M.-A. Madoui, S. Mangenot, C. Marchal, F. Maumus, J. Mercier, C. Michotey, O. Panaud, N. Picault, N. Rouhier, O. Rué, C. Rustenholz, F. Salin, M. Soler, M. Tarkka, A. Velt, A. Zanne, F. Martin, P. Wincker, H. Quesneville, A. Kremer, and J. Salse. 2018. Oak genome reveals facets of long lifespan. Nature Plants 4:440-452.

Roze, D. 2015. Effects of interference between selected loci on the mutation load, inbreeding depression, and heterosis. Genetics 201:745-757.

Roze, D., and A. Blanckaert. 2014. Epistasis, pleiotropy, and the mutation load in sexual and asexual populations. Evolution 86:137-149.

Roze, D., and R. Michod. 2010. Deleterious mutations and selection for sex in finite, diploid populations. Genetics 184:1095-1112.

Roze, D., and F. Rousset. 2005. Inbreeding depression and the evolution of dispersal rates: a multilocus model. The Amrican Naturalist 166:708-721.

Salguero-Gómez, R., O. R. Jones, E. Jongejans, S. P. Blomberg, D. J. Hodgson, C. MbeauAche, P. A. Zuidema, H. de Kroon, and Y. M. Buckley. 2016. Fast-slow continuum and reproductive strategies structure plant life-history variation worldwide. Proceedings of the National Academy of Sciences 113:230-235.

Schmid-Siegert, E., N. Sarkar, C. Iseli, S. Calderon, C. Gouhier-Darimont, J. Chrast, P. Cattaneo, F. Schütz, L. Farinelli, M. Pagni, M. Schneider, J. Voumard, L. Jaboyedoff, 
C. Fankhauser, C. Hardtke, L. Keller, J. Pannell, A. Reymond, M. Robinson-Rechavi, I. Xenarios, and P. Reymond. 2017. Low number of fixed somatic mutations in a longlived oak tree. Nature Plants 12:926-929.

Schoen, D., and S. Schultz. 2019. Somatic mutation and evolution in plants. Annual Review of Ecology, Evolution and Systematics 50:2.1-2.25.

Scofield, D., and S. Schultz. 2006. Mitosis, stature and evolution of plant mating systems: low- $\phi$ and high- $\phi$ plants. Proceedings of the Royal Society of London B 273:275-282.

Stearns, S. 1992. The Evolution of Life Histories. Oxford University Press.

Watson, J., A. Platzer, A. Kazda, S. Akimcheva, S. Valuchova, V. Nizhynska, M. Nordborg, and K. Riha. 2016. Germline replications and somatic mutation accumulation are independent of vegetative life span in arabidopsis. Proceedings of the National Academy of Sciences 113:12226-12231.

Weiner, J., L. Campbell, J. Pino, and L. Echarte. 2009. The allometry of reproduction within plant populations. Journal of Ecology 97:1220-1233.

West, G., J. Brown, and B. Enquist. 2001. A general model for ontogenetic growth. Nature 413:628-631.

Winn, A., E. Elle, S. Kalisz, P.-O. Cheptou, C. Eckert, C. Goodwillie, M. Johnston, D. Moeller, R. Sargent, and M. Vallejo-Marìn. 2011. Analysis of inbreeding depression in mixed-mating plants provides evidence for selective interference and stable mixed mating. Evolution 65:3339-3359. 


\section{FIGURE CAPTIONS WITH THEIR LABEL}

Figure 1. Demographic events over the course of one timestep. Deceased individuals are marked by a red cross, juveniles are depicted in light blue. Larger dots depict larger and older individuals.

Figure 2. Phenotypic and leading order fitness effect of a mutation. Top row: phenotypic effects on growth or survival are presented as a function of age, the dashed line depicts the mutant phenotype while the solid line depicts the wild type $(c=0.001, \varepsilon=0.01$ and $s=0.05$ for mutations affecting growth, and $S=0.99, s=0.005$ for mutations affecting survival). The dotted gray lines depict maximal size. Bottom row: Resulting effects of mutations on lifetime fitness as a function of life expectancy in a single locus case (black lines, $n=0$ ), and 10 mutations segregate at other loci (red lines, $n=10$ ). Note that the phenotypic effect of mutations $(s)$ differs between mutations affecting growth and survival.

Figure 3. Average number of mutations per haploid genome ( $n$, top row), inbreeding depression ( $\delta$, middle row), and mutation load ( $L$, bottom row) as a function of life expectancy $(E)$, for three selfing rates : $\alpha=0.1$ (blue), $\alpha=0.5$ (purple) and $\alpha=0.9$ (red). Each column corresponds to one type of mutation. Dots: averaged simulation results. Lines: analytical predictions accounting for genetic associations between loci using the LC approach (darker lines) and the LF approach (lighter lines). Parameters shown here are $\frac{c}{\varepsilon}=1, U=0.5, s=0.005, h=0.25$. Five replicates were run for each parameter set and results were averaged over all of them. Standard deviation bars are plotted but are hidden behind the dots depicting the averages. 
Figure 4. Equilibrium maintenance (left) and production (right) costs as function of life expectancy (log-scaled), for various selfing rates. Dots depict simulation results while lines depict analytical predictions. Parameters shown here are $c=\varepsilon=0.01, h=0.25$, $s=0.005$. 